\title{
Reconstructions of Noisy Digital Contours with Maximal Primitives Based on Multi-Scale/Irregular Geometric Representation and Generalized Linear Programming
}

\author{
Antoine Vacavant ${ }^{1}$, Bertrand Kerautret ${ }^{2}$, \\ Tristan Roussillon ${ }^{3}$, and Fabien Feschet $^{1}$ \\ 1 Université Clermont Auvergne, CNRS, SIGMA Clermont, Institut Pascal, F-63000 \\ Clermont- Ferrand, France, \\ antoine.vacavant@uca.fr \\ 2 LORIA, UMR CNRS 7503, Université de Lorraine, F-54506 Vandœuvre-lès-Nancy, \\ France, \\ bertrand.kerautret@univ-lorraine.fr \\ 3 Univ Lyon, INSA-LYON, LIRIS UMR 5205, F-69622, Villeurbanne, France, \\ tristan.roussillon@liris.cnrs.fr
}

\begin{abstract}
The reconstruction of noisy digital shapes is a complex question and a lot of contributions have been proposed to address this problem, including blurred segment decomposition or adaptive tangential covering for instance. In this article, we propose a novel approach combining multi-scale and irregular isothetic representations of the input contour, as an extension of a previous work [Vacavant et al., A Combined MultiScale/Irregular Algorithm for the Vectorization of Noisy Digital Contours, CVIU 2013]. Our new algorithm improves the representation of the contour by 1-D intervals, and achieves afterwards the decomposition of the contour into maximal arcs or segments. Our experiments with synthetic and real images show that our contribution can be employed as a relevant option for noisy shape reconstruction.
\end{abstract}

Keywords: Digital shape analysis, Irregular isothetic grids, Multi-scale analysis, Decomposition into maximal arcs, Decomposition into maximal segments

\section{Introduction}

The representation of digital contours is an important task in image analysis applications, since binary shapes obtained by image processing algorithms (preprocessing and segmentation) may be altered by noise. A lot of efforts have been made on these algorithms to produce smooth contours, by developing sophisticated deblurring and denoising algorithms [14], or by integrating regularization terms in segmentation process for instance [26]. However, these approaches significantly raise the computational complexity of the complete image analysis 
pipeline, and include other input-noise-dependent parameters to be tediously set for any new specific applications.

Hence, another approach consists in obtaining a faithful geometrical representation directly from any noisy digital contours. A lot of research works have addressed this question by fitting parametric curves (e.g. B-splines, rational Gaussian curves) to the input points $[4,8,10]$. These approaches require a parameter depending on input noise scale, in order to fit the objective function at best. In general, they do not use the fact that digital points belong to $\mathbb{Z}^{2}$, as this is always the case in the image plane.

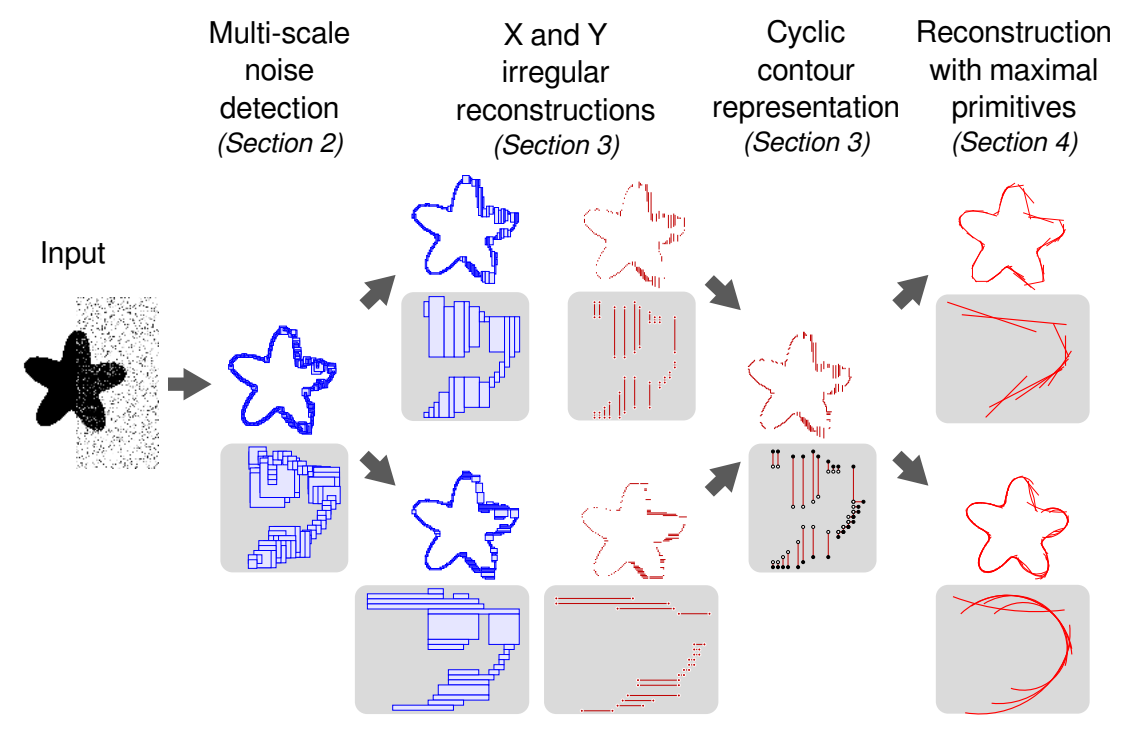

Fig. 1. Global pipeline of our approach. Input: a noisy contour. Output: maximal geometric primitives. Stages are: 1- Extraction of a multi-scale representation (unsupervised geometric noise detector); 2- Irregular isothetic representation (non overlapping cells) in $X$ and $Y$ directions; 3- 1-D intervals representation; 4- Fusion of the two directions to achieve a faithful geometric structure of the input contour.

In the digital geometry community, an important literature has been dedicated to this problem since the 80 's, by representing contours with several kinds of primitives (segments, arcs of circle). Thanks to theoretical concepts designed in digital geometry, these approaches extend the scheme of vectorization, wellknown in document analysis [3], consisting in converting pixels to line segments. In particular, some publications tackle the issue of fitting both straight segments and circular arcs to digital contours at the same time. The famous approach of Rosin and West [18] relies on least square fitting and is non parametric. Another parametric technique has been designed by Hilaire and Tombre [9], based on the notions of fuzzy digital segments and fuzzy digital arcs, and Faure and Feschet 
[5] by using $\alpha$-thick decomposition and combinatorial optimization. All of them are robust and accurate whilst the former two suffer from a high time complexity and are restricted to one pixel wide digital curves. Since multi-primitives decomposition can be viewed as a competition between primitives, the complexity can be tackled with an efficient and unified representation of the multiple decompositions with all individuals primitives. Relying on the work of Feschet and Tougne [6], each decomposition can be represented by a circular arc-graph in linear-time. Decomposition into several primitives can be solved in $\mathcal{O}(q n)$ where $q$ is the minimum number of intersecting primitives in the graph [2]. Other recent methods from state-of-the-art opt for different strategies, such as the adaptation of tangential cover [15] or the detection of dominant points [16].

In this article, we propose a novel unsupervised approach for reconstructing noisy digital contours by combining multi-scale and irregular isothetic representations as presented in Fig. 1. The complete pipeline of our approach works as follows. From an input (supposedly noisy) closed contour obtained from any image (first column), we first extract a multi-scale object, containing overlapping boxes, with an unsupervised geometric noise detector (second column). We then represent this structure by irregular isothetic objects that is with cells without any overlapping (third column). This is done by following two directions ( $X$ and $Y$ axes) simultaneously. Then these two $X$ and $Y$ axis aligned boxes are represented as lists of 1-D intervals between irregular cells (fourth column). Then, we combine both intervals to achieve a faithful geometric structure of the input contour using both $X$ and $Y$ oriented segments (fifth column) in a unified representation of the contour. At the end (sixth column) we compute maximal primitives within this last representation using the same Generalized Linear Programming approach for segments and arcs allowing us to produce decomposition of the contour into maximal straight line segments or circular arcs.

The article is organized as follows. In Section 2, we present the first step of our approach, aiming at representing the input contour as a multi-scale set of bounding boxes. These are then analyzed and converted into irregular isothetic structures, exposed in Section 3. Then, we describe the way to obtain decompositions into maximal primitives (Section 4), and experimental results with real and synthetic contours (Section 5) before concluding this article in Section 6 .

\section{Multi-Scale Noise Detection}

The noise level detection on a digital contour is an important problem, which can influence the quality of geometric estimators or contour representation algorithms. From the digital geometry domain, a method was proposed to automatically detect the amount of noise present on a digital structure [11]. This detection is based on the meaningful scale detection computed from asymptotic properties of the maximal segments. In particular, it is based on a theorem describing the evolution of the lengths of the maximal segments computed on the border of a shape on finer and finer grid sizes [13]. From such a multiscale analysis, the proposed algorithm consists in constructing, for each contour points $C$, a 

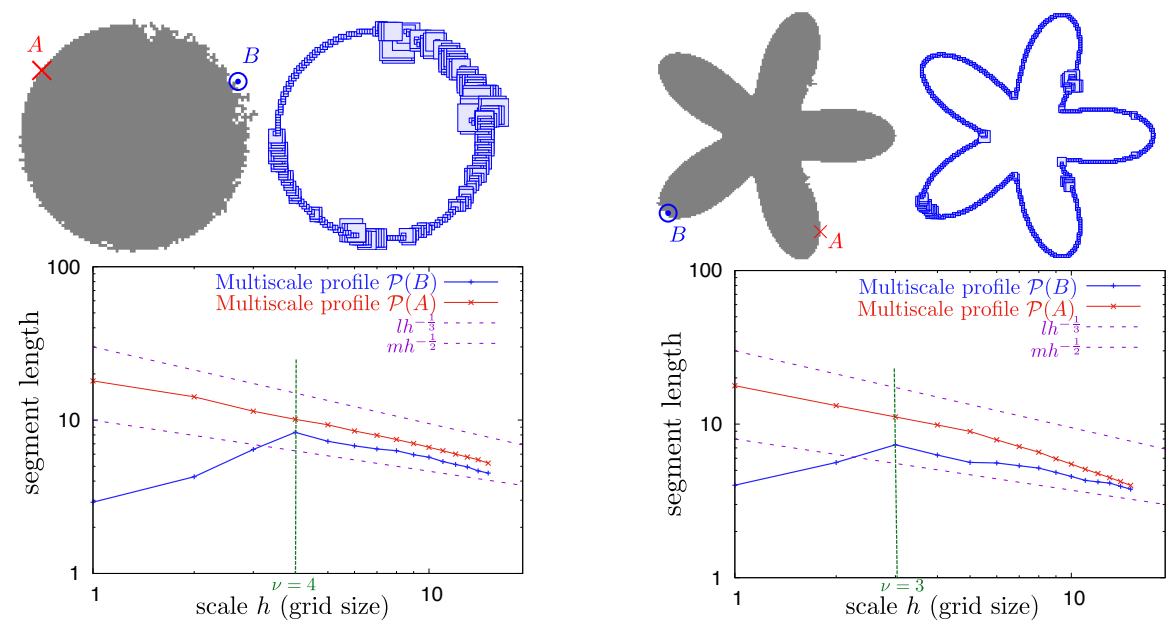

Fig. 2. Conversion of two noisy digital contours into meaningful scales (first row) and illustration of the multicale profiles for two points A and B (second row)

multiscale profile $(\mathcal{P}(C))$ defined by the segment length of all segments covering $P$ for larger and larger grid sizes $h$ (see graph of Fig. 2 second row). From each profile, the noise level is determined by the first scale $(n u)$ minus one for which the slope of $\mathcal{P}$ is decreasing and follows the awaited theoretical bounds between $h^{-\frac{1}{2}}$ and $h^{-\frac{1}{3}}$ if $C$ is on a non null curvature area and near $h^{-1}$ on flat part. On the examples of Fig. 2, the noise levels of points $\mathrm{A}$ are 0 since $\mathcal{P}(A)$ is always decreasing and $\mathrm{B}$ for the circle (resp. flower) has a noise level of 3 (resp. 3 ) since $\mathcal{P}(B)$ is increasing until scale 4 (resp. 3). This uncertainty can be represented as boxes and as exposed in Fig. 2 first row, a high noise in the contour will lead to a large box, and vice-versa. The algorithm can be tested on-line from any digital contour given by a netizen [12].

\section{Irregular Isothetic Cyclic Representation}

In this section, we first recall the $\mathbb{I}$-grid (Irregular Isothetic grid) model [22]:

Definition 1 (2-D $\mathbb{I}$-grid). Let $\mathcal{R}$ be a closed rectangular subset of $\mathbb{R}^{2}$. A 2-D $\mathbb{I}$-grid $G$ is a tiling of $\mathcal{R}$ with closed rectangular cells whose edges are parallel to the $X$ and $Y$ axes, and whose interiors have a pairwise empty intersection. The position of each cell $R$ is given by its center point $\left(x_{R}, y_{R}\right) \in \mathbb{R}^{2}$ and its length along $X$ and $Y$ axes by $\left(l_{R}^{x}, l_{R}^{y}\right) \in \mathbb{R}_{+}^{* 2}$.

This model permits to generalize many irregular image representations such as quad-trees, $k \mathrm{~d}$-trees, run-length encodings, and the geometry of frames encoded within video coding standards like MPEG, H.264, etc. For the rest of the article, we consider the following definitions for I-grids. 
Definition 2 (ve-adjacency and $e$-adjacency). Let $R_{1}$ and $R_{2}$ be two cells. $R_{1}$ and $R_{2}$ are ve-adjacent (vertex and edge adjacent) if :

$$
\text { or }\left\{\begin{array}{l}
\left|x_{R_{1}}-x_{R_{2}}\right|=\frac{l_{R_{1}}^{x}+l_{R_{2}}^{x}}{2} \text { and }\left|y_{R_{1}}-y_{R_{2}}\right| \leq \frac{l_{R_{1}}^{y}+l_{R_{2}}^{y}}{2} \\
\left|y_{R_{1}}-y_{R_{2}}\right|=\frac{l_{R_{1}}^{y}+l_{R_{2}}^{y}}{2} \text { and }\left|x_{R_{1}}-x_{R_{2}}\right| \leq \frac{l_{R_{1}}^{x}+l_{R_{2}}^{x}}{2}
\end{array}\right.
$$

$R_{1}$ and $R_{2}$ are e-adjacent (edge adjacent) if we consider an exclusive "or" and strict inequalities in the above ve-adjacency definition. The letter $k$ may be interpreted as e or ve in the following definitions.

A $k$-path from $R$ to $R^{\prime}$ is a sequence of cells $\left(R_{i}\right)_{1 \leq i \leq n}$ with $R=R_{1}$ and $R^{\prime}=R_{n}$ such that for any $i, 2 \leq i<n, R_{i}$ is $k$-adjacent to $R_{i-1}$ and $R_{i+1}$.

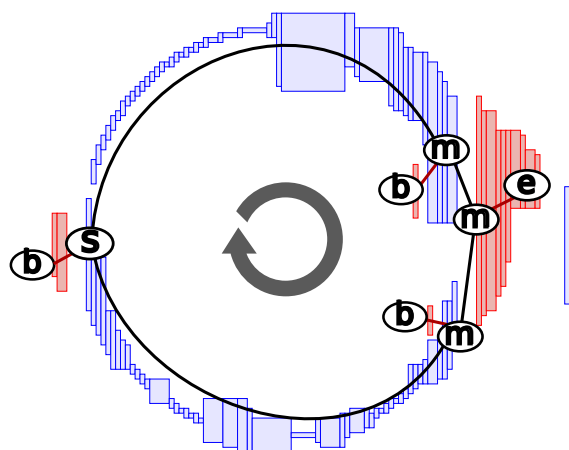

(a)

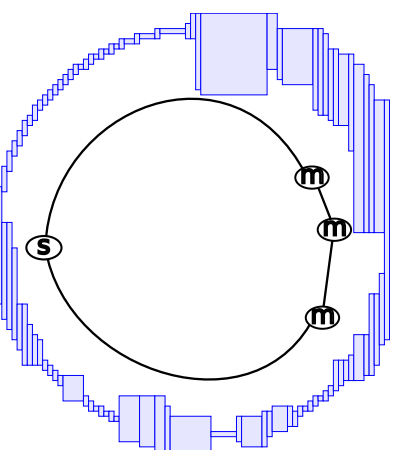

(b)

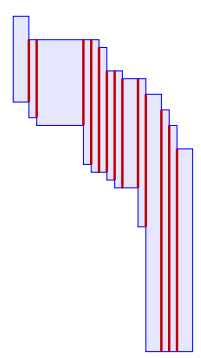

(c)

Fig. 3. From the meaningful scales of the Circle sample, we reconstruct a set of $k$-arcs converted to a $k$-curve thanks to the underlying graph (b). (c) presents a part of the cells obtained with intervals (red)

Definition 3 ( $k$-curve). Let $A=\left(R_{i}\right)_{1 \leq i \leq n}$ be a $k$-path from $R_{1}$ to $R_{n}$. Then $A$ is a $k$-curve iff each cell $R_{i}$ has exactly two $k$-adjacent cells in $A$.

As shown in Fig. 2, the meaningful boxes (denoted afterwards by the set $\mathcal{M}$ ) overlap and thus cannot be viewed as an irregular isothetic object directly (Definition 1). However each one contains a given number of pixels (at the initial resolution) so that the set of boxes $\mathcal{M}$ covers a subset of the input image. This subset $\mathcal{P}$, which is an irregular isothetic object, is transformed into $k$-arcs, i.e. open $k$-curves, and the respective adjacencies relations between arcs is represented by a Reeb graph structure [22], as illustrated in Fig. 3a [21, 23]. In that graph, each edge is associated to an irregular $k$-arc reconstructed. This process is driven by considering a given order relation, along $X$ or $Y$ axis (in this figure, $X$ axis has been chosen). With the support of the Reeb graph, we are then able to produce a cyclic representation of the contour by parsing $k$-arcs in a given order 
(e.g. clock-wise from the top-most element). We also remove extra branches of the graph, i.e. edges corresponding to $k$-arcs not belonging to the cycle (associated to red parts in Fig. 3a). In particular, graph edges comporting a node of degree 1 are removed. At the end of this process, we obtain a single $k$-curve (Fig. 3b), associated to a cyclic graph, and we consider the interface (Euclidean segment shared) between two consecutive cells in the $k$-curve, i.e. 1-D intervals.

By combining the intervals computed from both $X$ and $Y$ axes, we have thus two lists of segments representing the input contour, denoted by $\mathcal{S}_{X}$ and $\mathcal{S}_{Y}$, as shown in Fig. 4a. We set the internal and external points of these straight segments (black and white points in Fig. 4b) by considering the barycenter of the global shape as we did in [21].

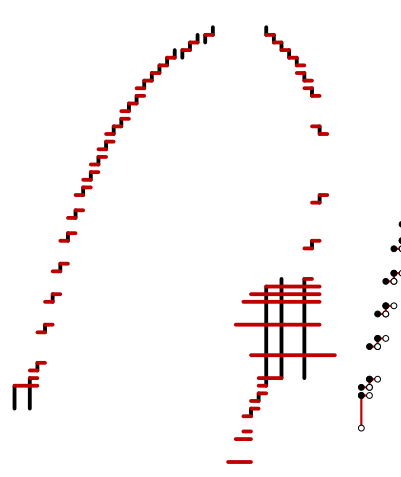

(a)

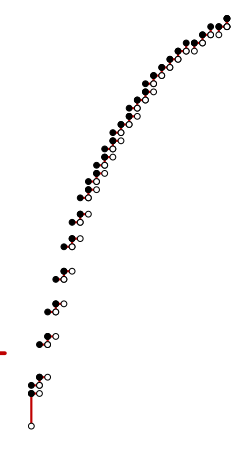

(b)

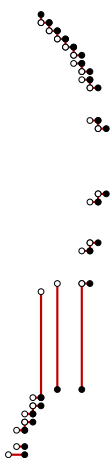

)

Fig. 4. A part of the two sets $\mathcal{S}_{X}$ (black) and $\mathcal{S}_{Y}$ (red) intervals from the Flower sample (a), and converted into a single set of intervals $\mathcal{S}_{X Y}$ (b). Internal points are dotted in white, external ones in black. We also illustrate some cases of our process, with specific examples of segments $s, s_{x}$ and $s_{y}$ (see text) in (c) and (d). In the images, red intervals are already in place in $\mathcal{S}_{X Y}$ and green ones are to be processed from $\mathcal{S}_{X}, \mathcal{S}_{Y}$

We then build a single list of intervals $\mathcal{S}_{X Y}=\left\{\mathcal{S}_{X Y}[i]\right\}_{1 \leq i \leq n}$ with $X$ - and $Y$ aligned elements by first removing segments from $\mathcal{S}_{Y}$ intersecting one or several ones in $\mathcal{S}_{X}$ (see again Fig. $4 \mathrm{a}$ and b). The converse choice can done (i.e. removing segments from $\mathcal{S}_{X}$ overlapping some of $\mathcal{S}_{Y}$ ), nevertheless, our option leads to a faithful representation of the input contour, as exposed later in experiments.

Then, we simultaneously parse the sets $\mathcal{S}_{X}$ and $\mathcal{S}_{Y}$ to construct this list by a linear and incremental approach, according to the size of these two lists. At an iteration of this process, consider the last segment added in $\mathcal{S}_{X Y}$, denoted by $s$, and the next segments to be added from $\mathcal{S}_{X}$ and $\mathcal{S}_{Y}$, denoted by $s_{x}$ and $s_{y}$ respectively. We add in $\mathcal{S}_{X Y}$ the closest interval from $s$. As an illustration, in Fig. $4 \mathrm{c}$, we add $s_{y}$, and in Fig. $4 \mathrm{~d}, s_{x}$.

During this process, adding segments of $\mathcal{S}_{X}$ and $\mathcal{S}_{Y}$ in $\mathcal{S}_{X Y}$ is realized in in $\mathcal{O}\left(\left|\mathcal{S}_{X}\right|+\left|\mathcal{S}_{Y}\right|\right)$. We can observe that we build a valid list of segments in $\mathcal{S}_{X Y}$, 
since two successive segments in the final list (not sharing the same end point) respect this condition:

$$
\overrightarrow{\mathcal{S}_{X Y}[i]} \cdot \overrightarrow{\mathcal{S}_{X Y}[(i+1) \bmod n]} \geq 0, \forall i=1, \ldots, n,
$$

wherein each segment is considered as a vector with the orientation given by internal and external endpoints. For instance, in Fig. $4 \mathrm{c}, \vec{s} \cdot \overrightarrow{s_{y}}=0$ and are added successively in $\mathcal{S}_{X Y}$, in Fig. $4 \mathrm{~d}, \vec{s} \cdot \overrightarrow{s_{x}}=0$, and any two consecutive parallel segments $\mathcal{S}_{X Y}[i]$ and $\mathcal{S}_{X Y}[i+1]$ will respect a strict positive dot product in Eq. 1. The validity of the list $\mathcal{S}_{X Y}$ also means that the list of internal points are ordered in the clockwise order, and follow the curvilinear abscissa of the input contour (and this is the same for the list of external points).

\section{Recognition of Straight Segments and Circular Arcs}

Even if the arrangement of straight segments in $\mathcal{S}_{X Y}$ is not completely random, it lacks regularity. For instance, the $X$-coordinates of the endpoints do not necessarily increase and for this reason, we cannot use the algorithm of O'Rourke [17] for the recognition of straight segments.

In this work, you use a general algorithm for the recognition of both straight segments and circular arcs, formulated as two instances of a Generalized Linear Programming (GLP) problem [1].

Our notations and definitions follow [1]. A GLP problem is a family $H$ of constraints and an objective function $\omega$ from subfamilies of $H$ to some totally ordered set $S$. In addition, $H$ and $\omega$ must be such that:

(C1) Monotonicity: $\forall F \subseteq G \subseteq H, \omega(F) \leq \omega(G)$,

(C2) Locality: $\forall F \subseteq G \subseteq H$ s.t. $\omega(F)=\omega(G)$ and for each $h \in H: \omega(F \cup h)>$ $\omega(F)$ iff $\omega(G \cup h)>\omega(G)$.

Note that the set $S$ must contain a special maximal element $\Omega$ so that $G \subseteq H$ is unfeasible if $\omega(G)=\Omega$ and feasible otherwise.

In our framework, the constraint set $H$ is given by the endpoints of the set of the $n$ straight segments of $\mathcal{S}_{X Y}$, with $n \geq 1$. Each straight segment has two endpoints: one with label "white", the other with label "black", as depicted in Fig. 4b. Let us denote the set of white (resp. black) endpoints by $P^{\circ}:=$ $\left\{p_{i}^{\circ}\right\}_{i=1 \ldots n}\left(\right.$ resp. $\left.P^{\bullet}:=\left\{p_{i}^{\bullet}\right\}_{i=1 \ldots n}\right)$. Let $\mathbb{P}($ resp. $\mathbb{D})$ be the set of all possible half-planes (resp. disks). For a given $\mathbb{X} \in\{\mathbb{P}, \mathbb{D}\}$, we want to find a shape $X \in \mathbb{X}$ that contains one point set, e.g. $P^{\circ}$, but not the other. In other words, we want to find $X \in \mathbb{X}$ under the constraint set $H:=\left\{h_{2 i-1}, h_{2 i}\right\}_{i=1 \ldots n}$, where

$$
\forall i=1, \ldots, n, h_{2 i-1}:=p_{i}^{\bullet} \in X, h_{2 i}:=p_{i}^{\circ} \notin X .
$$

The problem is unfeasible if it does not exist such a $X$, but feasible otherwise. In the latter case, we search for $X$ minimizing a given objective function.

For any $\mathbb{X} \in\{\mathbb{P}, \mathbb{D}\}$, there exists an objective function $\omega_{\mathbb{X}}$ so that $(\mathrm{C} 1)$ and $(\mathrm{C} 2)$ are true, which means that the above problem reduces to a GLP problem. 
The objective function $\omega_{\mathbb{D}}$ is chosen to either return $\Omega$ if the problem is unfeasible or the radius of a smallest separating disk for $H$ otherwise. By definition, the pair $\left(H, \omega_{\mathbb{D}}\right)$ satisfies the monotonicity condition $(\mathrm{C} 1)$, which coarsely says that the larger the constraint set is, the larger the smallest separating disk for this set is. In addition, since $n \geq 1$, the smallest separating disk, if it exists, is unique, which implies locality $(\mathrm{C} 2)$.

The objective function $\omega_{\mathbb{P}}$ returns $\Omega$ if the problem is unfeasible. Otherwise, the convex hulls of the point set to enclose and the point set to not enclose are well-defined because $n \geq 1$ and do not intersect. In this case, $\omega_{\mathbb{P}}$ returns the inverse of the minimal distance between the two convex hulls. The inverse is taken so that adding a non-redundant constraint makes the objective function increase. Again, the pair $\left(H, \omega_{\mathbb{P}}\right)$ satisfies conditions $(\mathrm{C} 1)$ and $(\mathrm{C} 2)$. As a result, depending on $\omega_{\mathbb{X}}$, we have to solve two different kinds of GLP problem.

There exists an easy-to-implement and randomized algorithm that solves these two kinds of GLP problem in expected linear-time [20]. It comes from the well-known randomized algorithm for the smallest enclosing circle problem [25]. It takes a pair $\left(H, \omega_{\mathbb{X}}\right)$ and returns a basis, ie. a minimal subfamily $B \in H$ such that $\omega_{\mathbb{X}}(B)=\omega_{\mathbb{X}}(H)$. The combinatorial dimension $d$ of the problem is the maximum size of any basis for any feasible family. For instance, $d=3$ for $\omega_{\mathbb{D}}$ $\left(\right.$ resp. $\omega_{\mathbb{P}}$ ) because at most three constraints uniquely define a disk (resp. the width between two convex polygons).

The algorithm is incremental and recursive. It may be coarsely described as follows. We iteratively add constraints. For each constraint, we check whether the new constraint violates the current basis or not. If yes, then we try to update the basis from the new constraint by recursively calling the same algorithm with all the previous constraints.

It is useful to have an on-line algorithm in order to compute the whole set of maximal segments [7] or arcs [19]. Since the original algorithm [20] is incremental, adding the constraints in order straightforwardly leads to an on-line algorithm. The drawback is that the random order can be used only in the recursive calls but not during the constraint discovery, which results to an increase of the expected time-complexity from linear to quadratic. However, we experimentally observe short running times. The next section shows results of our pipeline, employing this on-line algorithm for the reconstruction of maximal segments or arcs.

\section{Experimental Results}

We first present in Fig. 5 the whole set of maximal segments and arcs for the Flower image. Contrary to the previous work dedicated to pure vectorization [23], we do not calculate a unique polyline from a complex structure of $k$-arcs. Thanks to the cyclic irregular representation of the input contour, we are capable of reconstructing maximal primitives, bounded by 1-D intervals, whose lengths only depend on local input noise. Without any parameter, we obtain faithful representations of noisy shapes. Moreover, the results do not depend on any starting point, as it could be the case for other methodologies employing 


$$
\text { ** }
$$


greedy algorithms. Even in the case of an high amount of local noise, our algorithm successfully reconstructs sets of primitives, as illustrated in Fig. 5 (bottom) wherein the input digital contour is significantly corrupted and contains large discontinuities and holes (top-right of the shape). As in [21], we can also obtain the circle passing through the contour, by choosing maximal arcs (Fig. 5b).

We finally present the meaningful representation, and sets of maximal straight segments and circular arcs obtained with our algorithm, for two real images, in Fig. 6 (one contour) and Fig. 7 (two contours). The Char image leads to a noisy contour (Fig. 6b), which is accurately represented thanks to our algorithm. Maximal primitives (Fig. 6e,f) represent the complete contour, while one of our previous contributions (d) [23], an accurate vectorization by MLP (or Minimum Length Polyline), misses a part of the object, and produces abrupt angles in round parts. The Sign image $(350 \times 350$ pixels $)$ allows us to test the scalability of our method. The contours we have extracted generate a high number of meaningful boxes (1,364 boxes for external part, 1,234 for internal part) that we have processed without any extra effort.

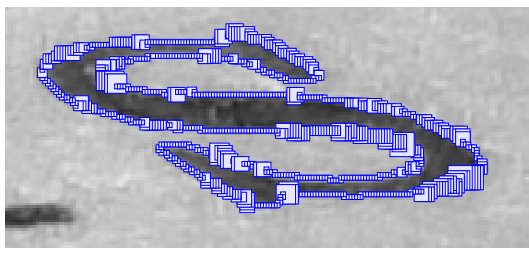

(a) Meaningful boxes

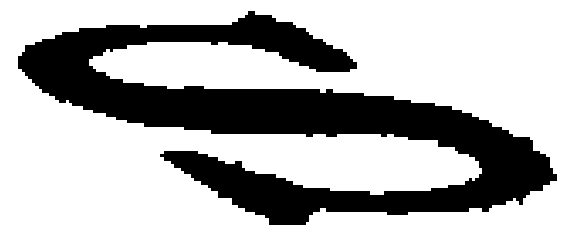

(b) Input contour

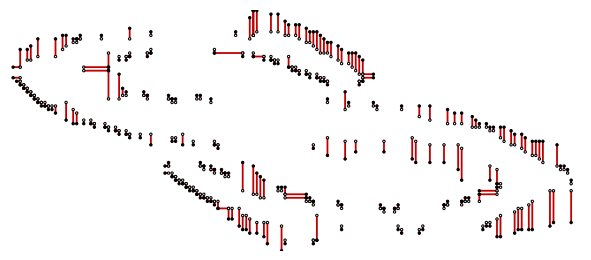

(c) 1-D intervals

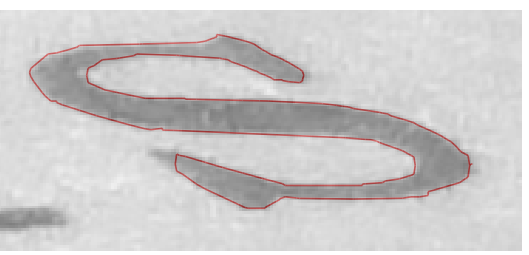

(d) Polyline (MLP)

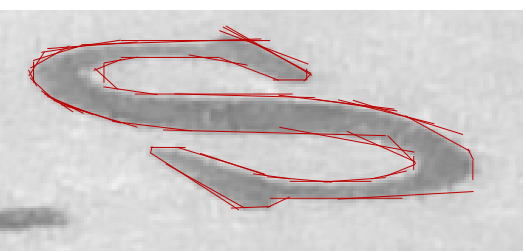

(e) Maximal segments

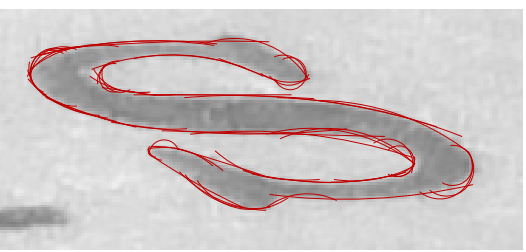

(f) Maximal arcs

Fig. 6. Results of our algorithm with the real image Char of size $185 \times 85$ pixels 


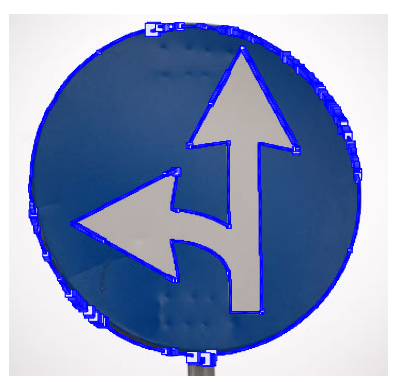

(a) Meaningful boxes

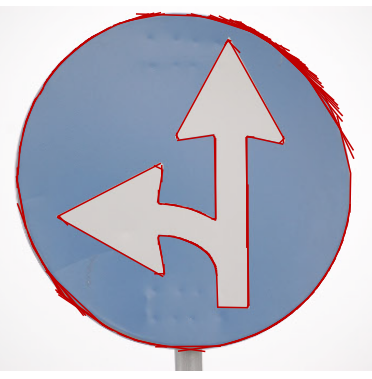

(b) Maximal segments

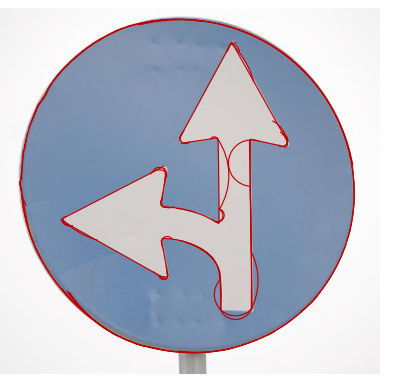

(c) Maximal circular arcs

Fig. 7. Results of our algorithm with the real image Sign of size $350 \times 350$ pixels

\section{Conclusion and Future Works}

In this article, we have proposed a novel approach combining multi-scale and irregular isothetic representations for the geometrical reconstruction of digital noisy contours. Our algorithm calculates a set of 1-D bounding intervals of the input shape, which permits to apply an on-line and incremental recognition algorithm. Our contribution has been successfully applied on synthetic and real images, encouraging us to exploit it in concrete image analysis contexts, and to investigate several lines of research.

Our first concern will consists in adapting the tangential cover approach [6] to our cyclic irregular representation. This will enable the calculation of a structure containing successive primitives, instead of overlapping maximal segments or arcs. Second, we would like to compare our contribution with other methods selected from state-of-the-art, e.g. [15, 16], and to test their robustness [24] with challenging data-sets of binary shapes, such as KIMIA. As a longer term, we plan to investigate the more general question of reconstructing digital shapes with other geometrical primitives, like B-splines and other parametric curves, with a similar framework we have presented herein.

\section{References}

1. N. Amenta. Helly-Type Theorems and Generalized Linear Programming. Disc. E6 Comp. Geom., 12(3):241-261, 1994

2. M.J. Atallah et al. An Optimal Algorithm for Shortest Paths on Weighted Interval and Circular-Arc Graphs, With Applications. Algorithmica, 14(5):429-441, 1995

3. H.S. Baird et al. Structured Document Image Analysis. Springer Verlag, 1992

4. P. Bo et al. A Graph-Based Method for Fitting Planar B-Spline Curves with Intersections. Journal of Comp. Des. and Eng., 3(1), 14-23, 2016

5. A. Faure and F. Feschet. Multi-primitive Analysis of Digital Curves. In IWCIA, LNCS 5852, pp. 30-42, 2009

6. F. Feschet and L. Tougne. On the Min DSS Problem of Closed Discrete Curves. Disc. App. Math., 151(1-3):138-153, 2005 
7. F. Feschet and L. Tougne. Optimal Time Computation of the Tangent of a Discrete Curve: Application to the Curvature. In DGCI, Springer LNCS 1568, pp. 31-40, 1999

8. A.A. Goshtasby. Fitting Parametric Curves to Dense and Noisy Points. In Int. Conf. on Curves and Surfaces, 1999

9. X. Hilaire and K. Tombre. Robust and Accurate Vectorization of Line Drawings. IEEE Transaction on Pattern Anal. and Machine Intel., 28(6):890-904, 2006

10. M. Karasalo et al. Contour Reconstruction Using Recursive Smoothing Splines Algorithms and Experimental Validation. Rob. and Aut. Sys., 57(6-7):617-628, 2009

11. B. Kerautret and J.O. Lachaud. Meaningful Scales Detection along Digital Contours for Unsupervised Local Noise Estimation. IEEE Transactions on Pattern Anal. and Machine Intel., 34(12):2379-2392, 2012

12. B. Kerautret and J.O. Lachaud. Meaningful Scales Detection: an Unsupervised Noise Detection Algorithm for Digital Contours. Image Proc. On Line, 4:98-115, 2014

13. J.O. Lachaud. Espaces Non-Euclidiens et Analyse d'Image : Modèles Déformables Riemanniens et Discrets, Topologie et Géométrie Discrète. Habilitation à Diriger des Recherches, Université Bordeaux 1, 2006 (en francais)

14. M. Lebrun et al. Secrets of Image Denoising Cuisine. Acta Num., 21:475-576, 2012

15. P. Ngo et al. , H. Nasser, I. Debled-Rennesson and B. Kerautret. Adaptive Tangential Cover for Noisy Digital Contours. In DGCI, LNCS 9647, pp. 439-451, 2016

16. T.P. Nguyen and I. Debled-Rennesson. Decomposition of a Curve into Arcs and Line Segments Based on Dominant Point Detection. In SCIA, Springer LNCS 6688, pp. $794-805,2011$

17. J. O'Rourke. An On-Line Algorithm for Fitting Straight Lines Between Data Ranges Com. of the ACM, 24(9):574-578, 1981

18. P.L. Rosin and G.A.W. West. Nonparametric Segmentation of Curves into Various Representations. IEEE Transactions on Pattern Anal. and Machine Intel., 17(12):1140-1153, 1995

19. T. Roussillon and J.O. Lachaud. Accurate Curvature Estimation along Digital Contours with Maximal Digital Circular Arcs. In DGCI, Springer LNCS 6636, pp. 43-55, 2011

20. M. Sharir and E. Welzl. A Combinatorial Bound for Linear Programming and Related Problems. In Annual Symp. on Theo. Aspects of Computer Science, Springer LNCS 577, pp. 567-579, 1992

21. J.-L. Toutant et al. Arc Recognition on Irregular Isothetic Grids and its Application to Reconstruction of Noisy Digital Contour. In DGCI, Springer LNCS 7749, pp. 265-276, 2013

22. A. Vacavant et al. Topological and Geometrical Reconstruction of Complex Objects on Irregular Isothetic Grids. In DGCI, Springer LNCS 4245, pp. 470-481, 2006

23. A. Vacavant et al. A Combined Multi-Scale/Irregular Algorithm for the Vectorization of Noisy Digital Contours. Comp. Vision and Image Und., 117(4):438-450, 2013

24. A. Vacavant. A Novel Definition of Robustness for Image Processing Algorithms. In IEEE ICPR, RRPR Workshop, Springer LNCS 10214, pp. 204-2015, 2016

25. E. Welzl. Smallest Enclosing Disks (Balls and Ellipsoids). New Results and New Trends in Comp. Science, 555(3075):359-370, 1991

26. O. Wirjadi. Survey of 3d Image Segmentation Methods. Berichte des Fraunhofer ITWM, 23, 2007 